Journal of Applied Pharmaceutical Science Vol. 5 (12), pp. 035-041, December, 2015

Available online at http://www.japsonline.com

DOI: $10.7324 / \mathrm{JAPS} .2015 .501206$

ISSN 2231-3354 (cc)) BY-NC-SA

\title{
Preliminary in vitro antimicrobial screening of chemical constituents isolated from the root of Lepleae mayombensis (Meliaceae)
}

\author{
Lazare Sidjui Sidjui $^{\mathrm{a}, \mathrm{b}, \mathrm{c}^{*}}$, Yvan Anderson Ngandjui Tchangoue ${ }^{\mathrm{d}}$, Sindhu Radhakrishnan ${ }^{\mathrm{c}}$, Perumal Karthiga ${ }^{\mathrm{e}}$, Paul \\ Djomgoue $^{\mathrm{h}}$, Rufin Marie Kouipou Toghueo ${ }^{\mathrm{f}}$, Louis Claire Ndel Famen ${ }^{\mathrm{g}}$, Gurumasy Annadurai ${ }^{\mathrm{c}}$, Gabriel Ngosong \\ Folefoc $^{\mathrm{b}}$
}

${ }^{a}$ Institute of Medical Research and Medicinal Plants Studies P.O Box 6163 Yaoundé, Cameroon. ${ }^{b}$ Department of Organic Chemistry, University of Yaoundé I, P.O Box 812, Yaoundé, Cameroon. 'Sri Paramakalyani Centre of Excellence in Enviromental Sciences Manonmaniam Sundaranar University, Alwarkurichi -627412, Tamil Nadu, India. ${ }^{\mathrm{d}}$ Department of chemistry, Higher Teachers Training College, P.O Box 47, Yaoundé, Cameroon. ${ }^{\mathrm{e} D e p a r t m e n t ~ o f ~}$ Chemistry, Manonmaniam Sudaranar University, Tirunelveli - 627012, Tamil Nadu, India. ${ }^{\mathrm{f}}$ Department of Biochemistry, University of Yaoundé I, P.O Box 812, Yaoundé, Cameroon. ${ }^{\mathrm{g}}$ Department of Pharmacy, Faculty of Medicine and Pharmaceutical Sciences, University of Douala, P.O Box: 2701 Douala, Cameroon. ${ }^{\mathrm{h}} \mathrm{TU}$ Chemnitz, Institut Für Anorganische Chemie, Straße Der Nationen 620911 Chemnitz, Germany.

\section{ARTICLE INFO}

Article history:

Received on: 18/09/2015

Revised on: 13/10/2015

Accepted on: 30/10/2015

Available online: 27/12/2015

\section{Key words:}

Leplaea mayombensis, Phytochemical study, Limonoids, Cycloartane antimicrobial.

\begin{abstract}
The Phytochemical study of Methanol/dichloromethane extract of root of Leplaea mayombenis led to the isolation and identification four limonoids: $(8 S, 9 R, 10 R, 11 S, 13 R, 17 R)$-6,11-dihydroxy-14,15-epoxy-4,4,8,10,13pentamethyl-17-(2-oxo-2,5-dihydrofuran-3-yl-)-11,12,13,15,16,17-hexahydro-4H-cyclopent $\alpha]$ phenanthrene$3,7(8 H, 9 H, 10 H, 14 H)$-dione (1), $(8 S, 9 R, 10 R, 11 S, 13 R, 17 R)-6,11$-dihydroxy-14,15-epoxy-4,4,8,10,13-pentamethyl -17-(23-methoxy-2-oxo-2,5-dihydrofuran-3-yl-)-11,12,13,15,16,17-hexahydro-4H-cyclopent [ $\alpha]$ phenanthrene 3,7 $(8 H, 9 H, 10 H, 14 H)$-dione (2), $(8 S, 9 R, 10 R, 11 S, 13 R, 17 R)$-6,11-dihydroxy-14,15-epoxy-17- $((s)$-2-hydroxy-5oxo-2,5-dihydrofurano-3-yl)-4,4,8,10,13-pentamethy-1,10,11,12,13,15,16,17-octahydro-4H-cyclopent $[\alpha]$ phenanthrene-3,7 $(8 H, 9 H, 10 H, 14 H)$-dione $\quad(3), \quad 8 S, 9 R, 10 R, 11 S, 13 R, 17 R)$-1,6,11-trihydroxy-14,15-epoxy-17[((2s)-2-hydroxy-5-oxo-2,5-dihydrofurano-3-yl)]-4,4,8,10,13-pentamethyl-1,10,11,12,13,15,16,17-octahydro- $4 H$ cyclopent $[\alpha]$ phenanthrene-3,7 $(8 H, 9 H, 10 H, 14 H)$-dione (4), one cycloartane : 9,10-cyclopropyl$(3 S, 5 R, 8 R, 13 R, 14 S, 17 R))-4,4,13,14,17-$ pentamethyl-17-((2R,5S,6R)-5,6,7-trihydroxy-6-methylheptan-2-yl)hexadecahydro- $1 H$-cyclopentane $[\alpha]$ phenanthrèn-3-ol (5) and three steroids: $\beta$-sitosterol (6), stigmasterol (7) and stigmasterol 3-O- $\beta$-D-glucopyranoside (8). Their structures were elucidated on the basis of spectroscopic analysis and by comparison of their spectral data with those reported in the literature. To the best of our knowledge, all these compounds were isolated for the first time from the Leplaea mayombensis. The antimicrobial studies showed that isolated compounds exhibit antimicrobial activity with inhibition zone diameters varying from 0.00 \pm 0.00 to $29.00 \pm 0.00 \mathrm{~mm}$ on both bacteria and fungi. 9,10-cyclopropyl-( $3 S, 5 R, 8 R, 13 R, 14 S, 17 R))-4,4,13,14,17$ pentamethyl-17-((2R,5S,6R)-5,6,7-trihydroxy-6-methylheptan-2-yl)-hexadecahydro-1 $\quad H$-cyclopentane[ $\alpha]$ phenanthrèn-3-ol (5) was the most active against Escherichia coli $(28.00 \pm 0.00 \mathrm{~mm})$, Pseudomonas sp $(27.00 \pm 0.00 \mathrm{~mm})$, Lactobacillus acidophilus $(29.00 \pm 0.00 \mathrm{~mm})$, Streptococcus pneumonia $(23.00 \pm 0.00 \mathrm{~mm})$ and Serratia entomophili $(29.00 \pm 0.00 \mathrm{~mm})$, C. albicans $(12 \pm 0.00 \mathrm{~mm})$, and $T$. viridae $(13 \pm 0.00 \mathrm{~mm})$ The results from this study support the conclusion that L. mayombensis contain many classes of antimicrobial compounds and therefore justify their traditional usage in the treatment of infectious diseases.
\end{abstract}

\section{INTRODUCTION}

Natural products are the most consistently successful source of drug leads. Natural products continue to provide greater structural diversity than standard combinatorial chemistry and so they offer major opportunities for finding novel low

\footnotetext{
* Corresponding Author

E-mail:lazaresidjui@yahoo.fr
}

molecular weight lead structures that are active against a wide range of assay targets (Harvey, 2000). Plants supply most of the active ingredients of traditional medicinal products, and plant extracts have long been used in screening programs in pharmaceutical companies and university institutes. It might be thought that most of the plant kingdom has been thoroughly examined in the search for biologically active molecules but only $10 \%$ of plants species existing around the world have been tested 
for some type of biological activity (Verpoorte, 1998). The genus Leplaea (meliaceae) has 8 species mainly widespread in tropical Africa. In Cameroon, 5 Leplaea species have been identified (Kouma et al., 1998). Significant chemical constituents of meliaceae family are triterpenoids and limonoids (Amit and Shailendra, 2006). Plants of meliaceae family are rich source of limonoids, which proves to be a class of bioactive natural products with highly oxygenated and modified triterpene skeleton (Li-Li et al., 2014). Limonoids are derivatives of terpenoids. meliaceous limonoids have attracted interest due to their diverse structures and biological activities, such as insect antifeedant, antimalarial, cytotoxic, and 11ß-HSD1 inhibitory effect (Tsamo et al., 2013; Nugroho et al., 2013; Rao et al., 2012; Tan et al., 2011).

In continuation of investigations of medicinal plants with aim to search for potent antimicrobial agents, the present study was designed to isolate compounds from the root of Leplaea mayombensis, and study their antibacterial and antifungal activities.

\section{MATERIALS AND METHODS}

\section{General experimental technique}

All organic solvents used were of analytical grade and Fractions were monitored by TLC and performed on precoated silica gel 60 F254 plates (Merck, Dramstadt, Germany). The spots were revealed using both ultra-violet light (254 $\mathrm{nm}$ and $366 \mathrm{~nm}$ ) and $10 \% \mathrm{H}_{2} \mathrm{SO}_{4}$ spray reagent. The structures of isolated compounds were elucidated by means of spectroscopic experiments mainly 1D and 2D NMR performed, on a 500 and 150 $\mathrm{MHz}$ Bruker Avance III-600 spectrometer equipped with a $5 \mathrm{~mm}$ $\mathrm{BBFO}+$ probe at $300 \mathrm{~K}$ and ESIMS / HRESIMS analyses recorded on a SYNAPT G2 HDMS (Waters) mass spectrometer and by comparison with literature data.

A Shimadzu HPLC, model LC-6AD, equipped with a Shimadzu SPD-6AV UV detector (detection UV $\lambda 217$ and 254) and a Shodex Asahipak GS-310 2Ga column (460 x 25 mm, $10 \mu \mathrm{m}$ particle size) was used for the analysis. For the column chromatography, Silica gel 60 (Acros Organics) and Sephadex LH 20 (Amersham Pharmacia Biotech AB) were used.

\section{Sample collection}

L. mayombensis root was harvested in YaoundéCameroon, (October, 2014) and identified by Mr. Victor Nana (Plant taxonomist) of the Cameroon National Herbarium (HNC), where a voucher specimens are deposited (46220/HNC). Then, root were collected, cut into small pecies, dried at room temperature and powdered.

\section{Extraction and isolation}

5 kilograms powdered roots were extracted by maceration in $\mathrm{MeOH}$ : $\mathrm{DCM}(1: 1 v / v)$ at room temperature. The filtrates were concentrated in rotavapor under reduce pressure to yield $450 \mathrm{~g}$ of extract (R). $300 \mathrm{~g}$ of this crude extract were subjected to fractionation by flash chromatography using $n$ - hexane, $n$ - hexane: AcOEt (3:1), $n$-hexane: AcOEt (1:1), AcOEt, and $\mathrm{MeOH}$ to yield five fractions: R1 (81.9 g), R2 (53.5 g), R3 $(40 \mathrm{~g}), \mathrm{R} 4$ (25.4 g) and R5 (71.9 g). Base on their TLC profile, fractions R1 and R2 were mixed together to yield fraction (R') and fractions R3 and R4 were mixed to obtain (R'). The three fractions R' (135.4 g), R', (65.4 g) and R5 (71.9 g) were used to follow the fractionation process. Fraction R' (50 g) was further subjected to column chromatography (CC) over silica gel $(4 \times 150 \mathrm{~cm}, 250 \mathrm{~g}, 70-230$ mesh) and eluted with $n$-hexane: AcOEt mixture with increasing polarity from $n$-hexane: AcOEt (9:1) to $n$-hexane: AcOEt (1:3).

Fractions obtained were subjected to other column chromatography with $n$-hexane- $\mathrm{CH}_{2} \mathrm{Cl}_{2}$ gradient mixtures. Eight compounds were obtained including; $\beta$-sitosterol $(6,12 \mathrm{mg})$, stigmasterol (7, $26 \mathrm{mg})$ and Stigmasterol 3-O- $\beta$-Dglucopyranoside (8, $27 \quad \mathrm{mg}), \quad 9,10$-cyclopropyl$(3 S, 5 R, 8 R, 13 R, 14 S, 17 R)-4,4,13,14,17$-pentamethyl-17 - $((2 R, 5 S, 6 R)$ -5,6,7-trihydroxy-6-methylheptan-2-yl)- hexadecahydro- $1 H$ cyclopentane $[\alpha]$ phenanthrèn-3-ol $\quad(5,7 \mathrm{mg}), \quad(8 S, 9 R, 10 R, 11 S$, $13 R, 17 R)-6,11$-dihydroxy-14,15 -epoxy-4,4,8,10,13 -pentamethyl17-(2-oxo-2,5-dihydrofuran-3-yl-)-11,12,13,15,16,17- hexahydro$4 H$-cyclopent $[\alpha]$ phenanthrene-3,7 $(8 H, 9 H, 10 H, 14 H)$-dione $(1,7$ $\mathrm{mg}), \quad(8 S, 9 R, 10 R, 11 S, 13 R, 17 R)-6,11$-dihydroxy-14,15-epoxy4,4,8,10,13 -pentamethyl -17-(23-methoxy-2-oxo-2,5dihydrofuran-3-yl-) -11,12,13,15,16,17- hexahydro-4 $H$ cyclopent $[\alpha]$ phenanthrene3,7 $(8 H, 9 H, 10 H, 14 H)$-dione $(2,5 \mathrm{mg})$, $(8 S, 9 R, 10 R, 11 S, 13 R, 17 R)$ - 6,11-dihydroxy-14, 15-epoxy-17- $((s)$ 2-hydroxy-5-oxo-2,5- dihydrofurano-3-yl) -4,4,8,10,13pentamethy-1,10,11,12,13,15,16,17 -octahydro- $4 H$ cyclopent $[\alpha]$ phenanthrene $-3,7(8 H, 9 H, 10 H, 14 H)$-dione $(3,7 \mathrm{mg})$ and $8 S, 9 R, 10 R, 11 S, 13 R, 17 R)$-1,6,11-trihydroxy-14,15-epoxy-17[((2s)-2-hydroxy-5-oxo-2,5-dihydrofurano-3-yl)] -4,4,8, 10,13pentamethyl-1,10,11,12,13,15,16,17-octahydro-4H-cyclopent $\quad[\alpha]$ phenanthrene $-3,7(8 H, 9 H, 10 H, 14 H)$-dione $(4,6 \mathrm{mg})$. All of the compounds were isolated for the first time from this plant.

\section{$(8 S, 9 R, 10 R, 11 S, 13 R, 17 R)-6,11-d i h y d r o x y-14,15$-epoxy-4,4,8, 10,13-pentamethyl-17-(2-oxo-2,5-dihydrofuran-3-yl-) $-\mathbf{1 1 , 1 2 , 1 3}$, $15,16,17$-hexahydro-4H-cyclopent $[\alpha]$ phenanthrene $3,7(8 H, 9 H$, 10H,14H)-dione (1) \\ ${ }^{1} \mathrm{H}$ NMR $(500 \mathrm{MHz}, \mathrm{CD} 5 \mathrm{CN}): \delta 7.25(1 \mathrm{H}, \mathrm{d}, 10.0 \mathrm{~Hz}$,} H-1), 7.10 (1H, s, H-22), $6.24(1 \mathrm{H}, \mathrm{d}, 10.0 \mathrm{~Hz}, \mathrm{H}-2), 5.03(1 \mathrm{H}, \mathrm{d}$, $6.3 \mathrm{~Hz}, \mathrm{H}-11), 4.71$ (2H, m, H-23), $4.08(1 \mathrm{H}, \mathrm{s}, \mathrm{H}-15), 3.02(1 \mathrm{H}$, m, H-17), 2.95 (1H, d, $15.1 \mathrm{~Hz}, \mathrm{H}-12 \mathrm{~b}), 2.85$ (1H, s, H-9), 2.26 (1H, dd, 15.1, $6.3 \mathrm{~Hz}, \mathrm{H}-12 \mathrm{a}), 2.14$ (1H, dd, 13.5, 6.5 Hz, H-16b), 2.04 (1H, m, H-16a), 1.88 (3H, s, H-28/H-29), 1.81 (3H, s, H-19), 1.78 (3H, s, H-30), 0.92 (3H, s, H-18) and ${ }^{13} \mathrm{C}$ NMR (150 MHz, CD5CN): 203.9 (C-3), 199.00 (C-7), 174.4 (C-21), 154.1 (C-1), 148.9 (C-22), 143.9 (C-6), 134.1 (C-5), 133.1 (C-20), 127.3 (C-2), 71.1 (C-23), 69.4 (C-14), 66.2 (C-11), 56.5 (C-15), 49.3 (C-4), 47.3 (C-8), 47.1 (C-12), 45.9 (C-9), 43.1 (C-17), 41.6 (C-13), 41.5 (C-10), 31.5 (C-16), 27.2 (C-28), 26.6 (C-19), 23.4 (C-30), 22.6 (C-18), 21.9 (C-29). 


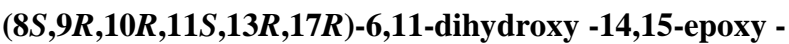
4,4,8,10, 13-pentamethyl- 17-(23-methoxy-2-oxo-2,5dihydrofuran-3-yl-) $-11,12,13,15,16,17-$ hexahydro4H-cyclopent $[\alpha]$ phenanthrene $3,7(8 H, 9 H, 10 H, 14 H)$-dione (2)

${ }^{1} \mathrm{H}$ NMR $(500 \mathrm{MHz}, \mathrm{CD} 5 \mathrm{CN}) \delta 6.39(2 \mathrm{H}, \mathrm{s}, \mathrm{H}-21)$, $6.08(1 \mathrm{H}, \mathrm{s}, \mathrm{H}-22), 4.87$ (1H, m, H-11), 4.02 (1H, m, H-15), 3.22 (1H, m, H-17), 2.81 (1H, dd, 18.8, 9.2 Hz, H-2a), $2.71(1 \mathrm{H}$, m, H-12b), 2.69 (1H, dd, 18.8, 9.6 Hz, H-2b), 2.51 (1H, s, H-9), $2.35(1 \mathrm{H}, \mathrm{dd}, 13.1,9.5 \mathrm{~Hz}, \mathrm{H}-1 \mathrm{~b}), 2.21(1 \mathrm{H}, \mathrm{m}, \mathrm{H}-12 \mathrm{a}), 2.11$ (1H, m, H-16b), 2.06 (1H, m, H-16a), 1.86 (1H, m, H-1a), 1.83 (3H, s, H-29), 1.75 (3H, s, H-28), 1.71 (3H, s, H-30), 1.60 (3H, s, $\mathrm{H}-19), 1.01$ (3H, s, H-18) and ${ }^{13} \mathrm{C}$ NMR (150 MHz, CD5CN): 214.8 (C-3), 199.3 (C-7), 171.4 ( C-23), 170.9 (C-20), 142.7 (C-6), 140.0 (C-5), 119.9 (C-22), 100.0 (C-21), 68.9 (C-13), 65.9 (C-11), 69.5 (C-13), 56.3 (C-14), 48.6 (C-9), 46.9 (C-8), 46.6 (C-12), 45.2 (C-16), 42.0 (C-12), 40.0 (C-10), 36.1 (C-1), 33.4 (C-2), 31.1 (C-15), 25.0 (C-28), 24.0 (C-30), 23.1 (C-18), 21.4 (C-29).

$(8 S, 9 R, 10 R, 11 S, 13 R, 17 R)-6,11-d i h y d r o x y-14,15-e p o x y-17-((s)-$ 2-hydroxy-5-oxo-2,5- dihydrofurano-3-yl) -4,4,8,10,13 pentamethy $-1,10,11,12,13,15,16,17-$ octahydro- $4 H$ cyclopent $[\alpha]$ phenanthrene $\mathbf{- 3 , 7}(\mathbf{8 H}, 9 \mathrm{H}, 10 \mathrm{H}, 14 \mathrm{H})$-dione(3)

${ }^{1} \mathrm{H}$ NMR (500 MHz, CD5CN) $\delta 6.44(1 \mathrm{H}, \mathrm{s}, \mathrm{H}-21), 6.10$ (1H, s, H-22), 4.92 (1H, m, H-11), 4.09 (1H, s, H-15), 3.25 (1H, m, H-17), 2.85 (1H, dd, 18.8, $9.2 \mathrm{~Hz}, \mathrm{H}-2 \mathrm{a}), 2.69$ (1H, dd, 18.8, 9.6 Hz, H-2b), 2.75 (1H, m H-12b) 2.56 (1H, s H-9), 2.35 (1H, dd, 13.2, 9.6 Hz, H-1b), 2.23 (1H, m, H12a), 2.16 (1H, m, H-16b), 2.07 (1H, m, H-16a), 1.89 (1H, m, H1a), 1.85 (3H, s, H-29), 1.77 (3H, s, H-29), 1.74 (3H, s, H-30), 1.61 (3H, s, H-19), 1.02 (3H, s, H-18) and ${ }^{13} \mathrm{C} \mathrm{NMR}(150 \mathrm{MHz}$, CD5CN):

214.9 (C-3), 199.5 (C-7), 171.6 (C-23), 170.8 (C-20), 142.9 (C-6), 140.0 (C-5), 120.0 (C-22), 100.4 (C-21), 69.7 (C-13), 66.1 (C-11), 69.7 (C-13), 56.5 (C-14), 48.8 (C-9), 47.0 (C-8), 46.7 (C-12), 45.4 (C-16), 42.1 (C-12), 40.1 (C-10), 36.2 (C-1), 33.6 (C-2), 31.5 (C15), 25.0 (C-28), 24.0 (C-30), 21.5 (C-29), 17.9 (C-18).

\section{$8 S, 9 R, 10 R, 11 S, 13 R, 17 R)-1,6,11-t r i h y d r o x y-14,15$-epoxy-17-} [((2s)-2-hydroxy-5-oxo-2,5-dihydrofurano-3-yl)] -4,4,8,10,13pentamethyl-1,10,11,12,13,15,16,17-octahydro $-4 H$ - cyclopent

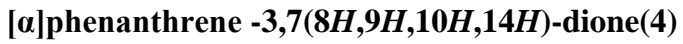

${ }^{1} \mathrm{H}$ NMR (500 MHz, CD5CN) $\delta 6.27(1 \mathrm{H}, \mathrm{s}, \mathrm{H}-21), 6.03$ (1H, s, H-22), 5.05 (1H, m, H-11), 4.68 (1H, m, H-1b), $4.13(1 \mathrm{H}$, m, H-15), 3.74 (1H, m, H-9), 3.25 (1H, m H-17), 3.21 (1H, m H2b), 3.14 (1H, m H-2a), 2.70 (1H, m, H-12b), 2.20 (1H, m, H-12a), 2.15 (1H, m, H-16b), 2.11 ( $3 \mathrm{H}, \mathrm{s}, \mathrm{H}-28), 2.06$ (1H, m, H-16a), 1.95 (3H, s, H-29), 1.82 (3H, s, H-30), 1.67 (3H, s, H-19), 1.07 (3H, s, H-18) and ${ }^{13} \mathrm{C}$ NMR (150 MHz, CD5CN): 214.9 (C-3), 199.6 (C-7), 171.6 (C-23), 170.8 (C-20), 145.3 (C-6), 138.4 (C-5), 119.9 (C-22), 100.3 (C-21), 71.1 (C-1), 70.0 (C-14), 66.6 (C-11), 56.6 (C-15), 49.0 (C-4), 46.8 (C-8), 46.5 (C-12), 45.5 (C-10), 45.4 (C-17), 45.0 (C-2), 42.2 (C-13), 40.4 (C-9), 25.0 (C-28), 24.1 (C-30), 22.9 (C-18), 22.1 (C-29), 18.1 (C-19).
9,10-cyclopropyl-(3S,5R,8R,13R,14S,17R)) -4,4,13,14,17pentamethyl-17-((2R,5S,6R)-5,6,7-trihydroxy-6-methylheptan -2-yl)-hexadecahydro-1H-cyclopentane[ $\alpha]$ phenanthrèn -3-ol (5).

${ }^{1} \mathrm{H}$ NMR $(500 \mathrm{MHz}, \mathrm{CD} 5 \mathrm{CN}) \delta 4.31(1 \mathrm{H}, \mathrm{d}, 10.6 \mathrm{~Hz}, \mathrm{H}-$ 26a), 4.17 (1H, m, H-24), 4.11 (1H, d, $10.6 \mathrm{~Hz}, \mathrm{H}-26 \mathrm{~b}), 3.53(1 \mathrm{H}$, dd, 4.5, 11.5 Hz, H-3), 2.13 (1H, m, H-15b), 2.01 (1H, m, H-11a), 2.00 (1H, m, H-2a), 1.98 (1H, m, H-23a), 1.97 (1H, m, H-16a), 1.87 (1H, m, H-23b), 1.80 (1H, m, H-22a), 1.71 (1H, m, H-22b), 1.66 (1H, m, H-16b), 1.97 (1H, m, H-23a), 1.64 (3H, m, H-27), 1.61 (2H, m, H-12), 1.57 (1H, m, H-6a), 1.55 (1H, m, H-20), 1.52 (1H, m, H-1a), 1.48 (1H, dd, 4.6, 12.3, H-8), 1.34 (1H, m, H-2b), 1.32 (1H, m, H-5), 1.31 (1H, m, H-7b), 1.27 (1H, m, H-15a), 1.25 (1H, m, H-1b), 1.24 (3H, m, H-28), 1.14 (1H, m, H-11b), 1.11 (3H, m, H-29), 1.02 (3H, m, H-21), $1.00(1 \mathrm{H}, \mathrm{m}, \mathrm{H}-7 \mathrm{a}), 1.00(1 \mathrm{H}$, m, H-1b), 1.00 (1H, s, H-17), 1.00 (3H, m, H-18), 0.90 (3H, s, H30), 0.78 (1H, m, H-6b), 0.53 (1H, m, H-19a), 0.30 (1H, m, H$19 \mathrm{~b})$ and ${ }^{13} \mathrm{C}$ NMR (150 MHz, CD5CN): $78.9(\mathrm{C}-3), 76.6(\mathrm{C}-24)$, 75.1 (C-25), 69.5 (C-26), 53.3(C-17), 49.5 (C-14), 48.5 (C-8), 47.7 (C-5), 45.7 (C-13), 41.3 (C-4), 36.5 (C-21), 36.2 (C-15), 34.5 (C22), 33.5 (C-12), 32.7 (C-1), 31.5 (C-23), 30.5 (C-19), 28.7 (C16/C-2), 27.0 (C-11), 26.8 (C-10), 26.5 (C-7), 26.3 (C-2869.5 (C26), 21.6 (C-6), 20.5 (C-27), 20.3 (C-9), 19.7 (C-30), 18.7 (C-18), 15.5 (C-29).

\section{$\beta$-Sitosterol (6)}

${ }^{13} \mathrm{C}$ NMR (150 MHz, CDCl3): 837.24 (C- 1), $31.66(\mathrm{C}-$ 2), 71.81 (C-3), 42.31 (C-4), 140.75 (C-5), 121.72 (C- 6), 31.90, 31.89 (C-7, C-8), 50.12 (C-9), 36.14 (C-10), 21.07 (C- 11), 39.76 $(\mathrm{C}-12), \quad 42.31 \quad(\mathrm{C}-13), \quad 56.75 \quad(\mathrm{C}-14), \quad 24.30 \quad(\mathrm{C}-15), \quad 28.24$ (C-16), 56.04 (C-17), 11.85 (C-18), 19.39 (C-19), 36.49 (C-20), 19.02 (C-21), 33.93 (C-22), 29.13 (C-23), 45.82 (C-24), 26.04 (C- 25), 18.77 (C-26), 19.81 (C-27), 23.05 (C-28), 11.97 (C29).

\section{Stigmasterol (7)}

${ }^{13} \mathrm{C}$ NMR (150 MHz, CDCl3): $\delta 37.24(\mathrm{C}-1), 31.66(\mathrm{C}-$ 2), 71.81 (C-3), 42.30 (C-4), 140.75 (C-5), 121.72 (C- 6), 31.89, 31.90 (C-7, C-8), 50.12 (C-9), 36.49 (C-10), 21.07 (C- 11), 39.67 $(\mathrm{C}-12), \quad 42.20 \quad(\mathrm{C}-13), \quad 56.75 \quad(\mathrm{C}-14), 24.35 \quad$ (C-15), 28.91 (C-16), 55.94 (C-17), 12.04 (C-18), 19.39 (C-19), 40.49 (C-20), 21.07 (C-21), 138.31(C-22), 129.26 (C-23), 51.23 (C-24), 31.89 (C-25), 21.20 (C-26), 18.97 (C-27), 25.40 (C-28), 12.25 (C-29).

\section{Stigmasterol 3-O- $\beta$-D-glucopyranoside (8)}

13C NMR (150 MHz, C5D5N): $\delta \mathrm{C} 141.1$ (C-5), 139.0 (C-22), 129.6 (C-23), 122.1 (C-6), 102.8 (C-1'), 78.8 (C-3'), 78.7 (C-5'), 78.3 (C-3), 75.5 (C-2'), 71.9 (C-4'), 63.0 (C-6'), 57.0 (C14), 56.4 (C-17), 51.6 (C-24), 50.5 (C-9), 42.6 (C-13), 41.0 (C20), 40.1 (C-12), 39.5 (C-4), 37.7 (C-1), 37.1 (C-10), 32.4 (C-7), 32.2 (C-8, C-25), 30.4 (C-2), 29.6 (C-16), 25.9 (C-28), 24.7 (C15), 21.7 (C-21), 21.5 (C-11, C-26), 19.6 (C-19), 19.4 (C-27), 12.7 (C-29), 12.3 (C-18). 


\section{Phytochemical screening of plant extract and fractions}

Crude extract and fractions were subjected to phytochemical screening to detect the presence of alkaloids, tannins, saponins, triterpenoides, steroids, flavonoids, phenol, coumarins and antraquinones using protocols described by Harbone, 1973.

\section{Test microorganisms}

The test microorganisms includes: Gram-positive bacteria (Streptococcus pneumonia, Entrecoccus coaccae and Lactobacillus acidophilus), Gram-negative bacteria (Escherichia coli, Serratia entomophilia and Pseudomonas sp.) and fungal strains (Aspergillus flavus, Trichophyton viridae and Candida albicans) obtained from the Sri Paramakalyani Centre of Excellence in Enviromental Sciences Manonmaniam Sundaranar University, Alwarkurichi -627412, Tamil Nadu, India.

\section{Stock solutions and disc preparation}

For the antimicrobial activity, stock solutions of compounds were prepared at $0.02 \mathrm{mg} / \mathrm{mL}$ in DMSO $10 \%$. Amplicillin, Chloramphenicol, Tetracyclin, Fluconazole and Nystatin were prepared in the same conditions. For disc preparation, $10 \mu \mathrm{L}$ of each stock solution was dropped onto sterilized paper disks $(6 \mathrm{~mm}$ diameter $)$ and dried at room temperature for a final concentration of $0.02 \mathrm{mg} /$ disc.

\section{Antimicrobial activity}

In vitro antimicrobial activity was assess by disc diffusion method using Mueller Hinton (Bauer et al., 1966) Agar (MHA) and Sabouraud dextrose Agar (SDA) obtained from Mast Group Ltd. The Agar plates were prepared by pouring $15 \mathrm{~mL}$ of molten media into sterile plates $(90 \mathrm{~mm})$. The plates were allowed to solidify for $5 \mathrm{~min}$ and $0.1 \mathrm{~mL}$ of inoculum suspension was swabbed uniformly and the inoculum allowed drying for 5 minutes.

The different compounds and references drugs loaded at $0.02 \mathrm{mg} / \mathrm{discs}$ were placed on the surface of the medium and allowed to diffuse for $5 \mathrm{~min}$. The plates were incubated at $35{ }^{\circ} \mathrm{C}$ for 24 hours for bacteria and for 48 hours for fungi. Negative control was prepared using $10 \%$ DMSO. At the end of incubation, inhibition zones formed around the disc were measured with a Vernier Calliper in millimeter. Each experiment was performed in triplicate.

\section{RESULTS AND DISCUSSION}

\section{Phytochemical content of crude extract and fractions}

The preliminary phytochemical study (Table 1) showed that extract from root of L. mayombensis contain phenols, steroids, terpenoids, flavonoids, coumarins, alkaloids, saponins and tannins. The same chemical composition was found with methanol fraction. Only alkaloids were absent in ethyl acetate fraction of this extract. Whereas, hexane fraction contains only terpenoids, steroids and coumarins.
Table 1: Phytochemical composition of extracts and fractions of $L$. mayombensis

\begin{tabular}{lcccc}
\hline \multirow{2}{*}{ Chemical class } & Crude extract & \multicolumn{3}{c}{ Fractions } \\
\cline { 2 - 5 } & M-C & H-F & AE-F & M-F \\
\hline Alkaloids & + & - & - & ++ \\
Anthraquinones & + & - & ++ & ++ \\
Flavonoids & ++ & - & ++ & ++ \\
Phenols & ++ & - & ++ & ++ \\
Saponins & ++ & - & + & ++ \\
Sterols & ++ & ++ & ++ & ++ \\
Tanins & ++ & - & + & ++ \\
Terpenoids & ++ & ++ & ++ & ++ \\
Coumarins & ++ & + & ++ & ++ \\
\hline +: Slight presence, ++: Heavy presence, -: Absence; $\mathrm{M}-\mathrm{C}: \mathrm{MeOH}_{-} \mathrm{CH}_{2} \mathrm{Cl}$ & $(1: 1$, \\
$v / v)$ crude extract; H-F: Hexane fraction, AE-F: ethyl acetate fraction, $\mathrm{M}-\mathrm{F}:$ \\
Methanol fraction.
\end{tabular}

\section{Fractionation and isolation of compounds}

Fractionation of $\mathrm{MeOH} / \mathrm{DCM}$ root extract of $L$. mayombensis by column chromatography lead to isolation and purification of eight compounds (1-8). The structures of the isolated compounds were determined by spectroscopic analysis, especially, ${ }^{1} \mathrm{H}$, and ${ }^{13} \mathrm{C}$ NMR spectra in conjunction with $2 \mathrm{D}$ experiments, COSY, HSQC, HMBC and direct comparison with reference data from available literature (Figure 1).

They are: $(8 S, 9 R, 10 R, 11 S, 13 R, 17 R)-6,11$-dihydroxy-14,15-epoxy4,4,8,10,13-pentamethyl-17-(2-oxo-2,5-dihydrofuran-3-yl-) -11, 12,13,15,16,17-hexahydro-4H-cyclopent [ $\alpha]$ phenanthrene- $\quad 3,7$ $(8 H, 9 H, 10 H, 14 H)$-dione (1) (Luo et al., 2000, Vieira et al., 2013, Kai-Long et al., 2014), (8S,9R,10R,11S,13R,17R)-6,11-dihydroxy14,15-epoxy-4,4,8,10,13-pentamethyl-17-(23-methoxy-2-oxo-2,5dihydrofuran-3-yl-)-11,12,13,15,16,17-hexahydro-4H- cyclopent [ $\alpha]$ phenanthrene 3,7(8H,9H,10H,14H)-dione (2) (Luo et al., 2000, Vieira et al., 2013, Kai-Long et al., 2014), $(8 S, 9 R, 10 R, 11 S, 13 R, 17 R)-6,11$-dihydroxy-14,15-epoxy-17- $((s)-2$ hydroxy-5-oxo-2,5-dihydrofurano-3-yl)-4,4,8,10,13- pentamethy$1,10,11,12,13,15,16,17$-octahydro- $4 H$-cyclopent $[\alpha]$ phenanthrene 3,7(8H,9H,10H,14H)-dione (3) (Luo et al., 2000, Vieira et al., 2013, Kai-Long et al., 2014), 8S,9R,10R,11S,13R,17R)-1,6,11trihydroxy-14,15-epoxy-17- $\quad[((2 s)$-2-hydroxy-5-oxo-2,5dihydrofurano-3-yl)]-4,4,8,10,13-pentamethyl- $\quad 1,10,11,12,13,15$, 16, 17-octahydro- $4 H$-cyclopent $\alpha]$ phenanthrene $-3,7(8 H, 9 H, 10 H$ ,14H)-dione (4) (Luo et al., 2000, Vieira et al., 2013, Kai-Long et al., 2014); 9,10-cyclopropyl-(3S,5R,8R,13R,14S,17R))4,4,13,14,17-pentamethyl-17-((2R,5S,6R)-5,6,7- trihydroxy-6methylheptan-2-yl)-hexadecahydro-1 $\quad H$-cyclopentane $[\alpha]$ phenanthrèn-3-ol (5) (Luo et al., 2000, Vieira et al., 2013, KaiLong et al., 2014), steroids yields $\beta$-sitosterol (6) (Tagatsing et al., 2011, Rao et al., 2012, Khalijah et al., 2012, Tchouya et al., 2013), stigmasterol (7) (Tagatsing et al., 2011, Rao et al., 2012, Khalijah et al., 2012, Tchouya et al., 2013)and stigmasterol 3-O- $\beta$ D-glucopyranoside (8) (Tagatsing et al., 2011, Rao et al., 2012, Khalijah et al., 2012, Tchouya et al., 2013). Our results represent the first report of isolation of constituents 1-8 from $L$. mayombensis. Moreover, these compounds have been found in others plants species such as Walsura yunnanensis, Walsura trifoliata (Luo et al., 2000, Vieira et al., 2013, Kai-Long et al., 2014) 


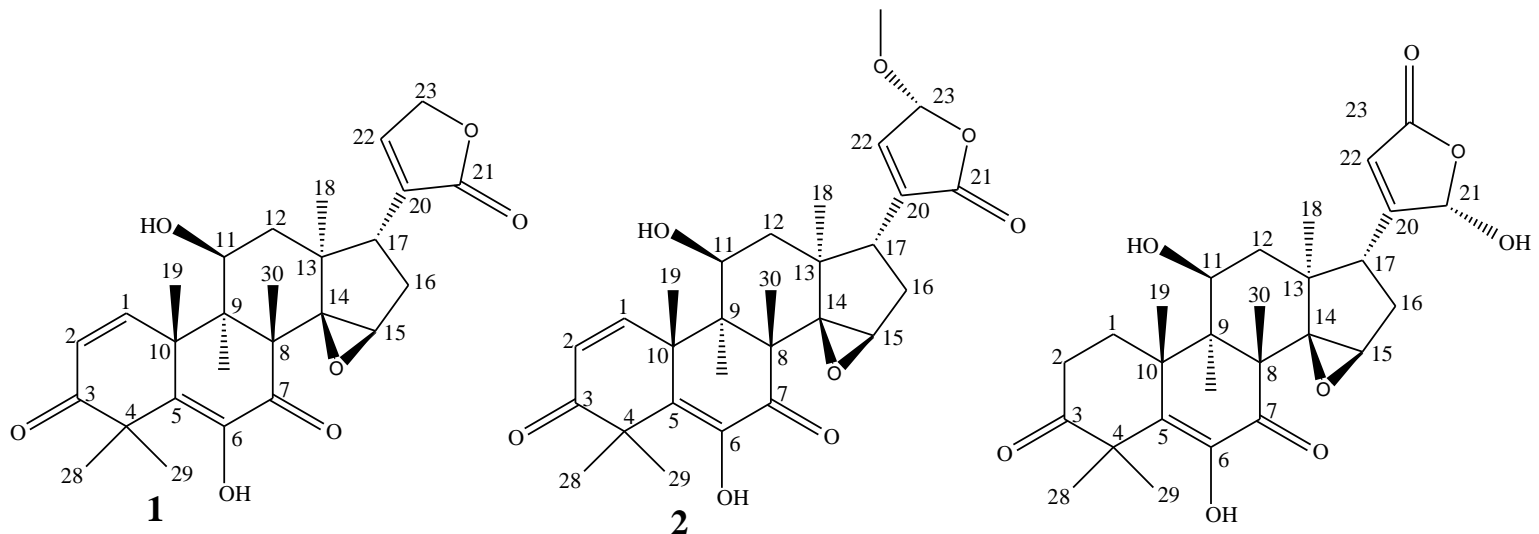

3

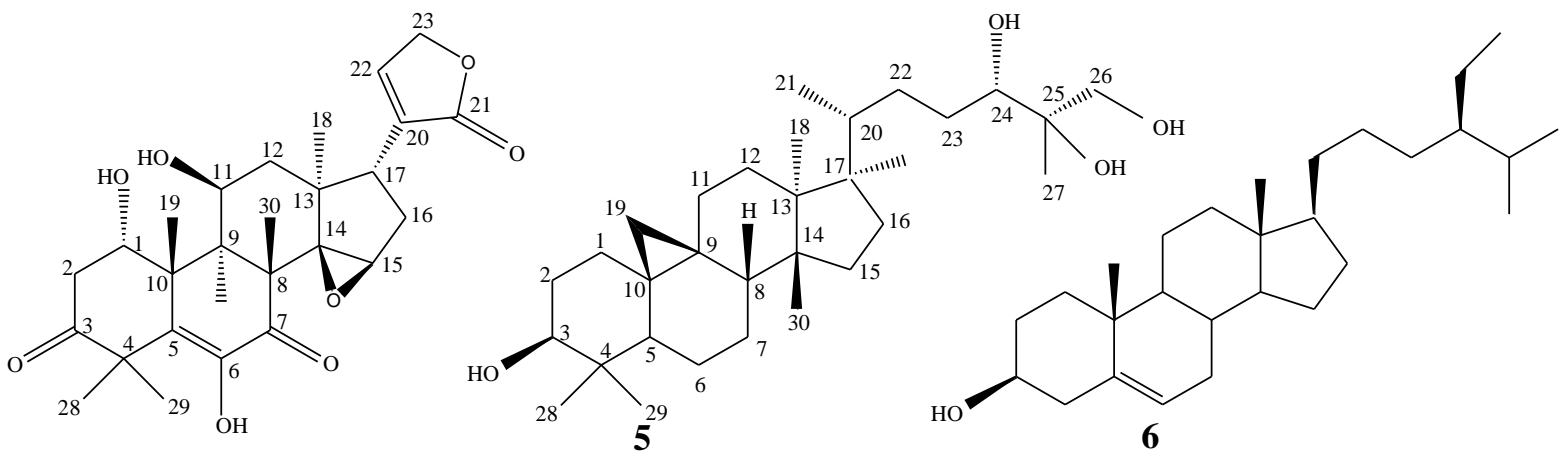

4

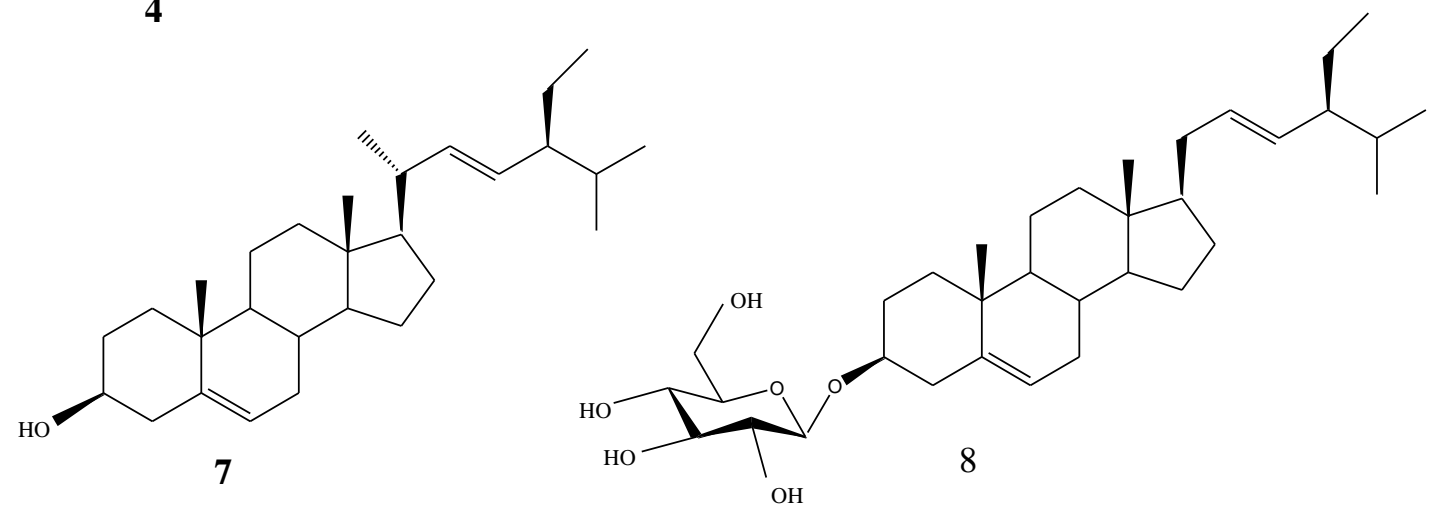

Fig. 1: Compounds isolated from roots of L. mayombensis.

\section{Antimicrobial activities}

The bacterial inhibition zone diameters of crude extracts, fractions and compounds are summarized in table 2 . The results below indicate that the inhibition zone diameters vary from $0.00 \pm$ 0.00 to $29.00 \pm 0.00 \mathrm{~mm}$ on both bacteria and fungi. This inhibition was found to depend to compounds and microorganism tested. $\quad(8 S, 9 R, 10 R, 11 S, 13 R, 17 R)-6,11$-dihydroxy-14,15-epoxy4,4,8,10,13-pentamethyl-17-(2-oxo-2,5-dihydrofuran-3-yl-)-11,12, $13,15,16,17$-hexahydro- $4 H$-cyclopent $[\alpha]$ phenanthrene-3,7 $(8 H, 9 H$, $10 H, 14 H$ )-dione (1) was found to be ineffective against bacteria strains but showed moderate inhibition (11-12 mm) against fungal strains. $\beta$-sitosterol (6) were active only against two gram negative (9 $\mathrm{mm}$ ) bacteria (S. entomophilia and Pseudomonas sp) and filamentous fungi $(10 \mathrm{~mm})(A$. flavus and $T$ viridae). Stigmasterol (7) was active against one gram negative and one filamentous fungus $(9 \mathrm{~mm})$. Compounds 8 inhibit the growth $(9-10 \mathrm{~mm})$ of two gram negative, one gram positive and one filamentous fungus. The good activity was observed with broad spectrum antimicrobial 9,10-cyclopropyl-(3S,5R,8R,13R,14S,17R)4,4,13,14,17-pentamethyl-17-((2R,5S,6R)-5,6,7- trihydroxy- 6methylheptan-2-yl)-hexadecahydro-1 $H$ - cyclopentane $[\alpha]$ phenanthrèn- 3-o19,10-cyclopropyl-(3S,5R,8R,13R,14S,17R) )4,4,13,14,17-pentamethyl-17-((2R,5S,6R)-5,6,7 -trihydroxy-6methylheptan-2-yl)-hexadecahydro-1 $\quad H \quad$-cyclopentane $[\alpha]$ phenanthrèn-3-ol (5) which were the most active against both bacteria, yeast and filamentous fungi with inhibition diameter ranging from 12 to $29 \mathrm{~mm}$.

All the compounds tested have showed antimicrobial activities on at least one microorganism. This activity was better than those exhibits by referents antibiotics. 
Table 2: Inhibition zone diameter of compounds 1, 5, 6-8 isolated from root of L.mayombensis.

\begin{tabular}{|c|c|c|c|c|c|c|c|c|c|c|}
\hline \multirow[t]{3}{*}{ Microoganisms } & \multicolumn{10}{|c|}{ Inhibition zone diameter $(\mathbf{m m} \pm$ SD) } \\
\hline & \multicolumn{5}{|c|}{ Compounds (0.02mg/dic) } & \multicolumn{5}{|c|}{ Reference(Positive control) } \\
\hline & 1 & 5 & 6 & 7 & 8 & Amp & Chl & Tet & Flu & Nys \\
\hline S.entomophilia & $0.00 \pm 0.00$ & $19.00 \pm 0.00$ & $9.00 \pm 0.00$ & $0.00 \pm 0.00$ & $10.00 \pm 0.00$ & $15.00 \pm 0.00$ & $38.00 \pm 0.00$ & $31.00 \pm 0.00$ & nd & nd \\
\hline E.caccae & $0.00 \pm 0.00$ & $13.00 \pm 0.00$ & $0.00 \pm 0.00$ & $0.00 \pm 0.00$ & $10.00 \pm 0.00$ & $13.00 \pm 0.00$ & $40.00 \pm 0.00$ & $6.00 \pm 0.00$ & nd & nd \\
\hline P. $s p$ & $0.00 \pm 0.00$ & $27.00 \pm 0.00$ & $9.00 \pm 0.00$ & $9.00 \pm 0.00$ & $9.00 \pm 0.00$ & $28.00 \pm 0.00$ & $28.00 \pm 0.00$ & $28.00 \pm 0.00$ & nd & nd \\
\hline E. coli & $0.00 \pm 0.00$ & $28.00 \pm 0.00$ & $0.00 \pm 0.00$ & $0.00 \pm 0.00$ & $0.00 \pm 0.00$ & $0.00 \pm 0.00$ & $17.00 \pm 0.00$ & $12.00 \pm 0.00$ & nd & nd \\
\hline L.acidophilus & $0.00 \pm 0.00$ & $29.00 \pm 0.00$ & $0.00 \pm 0.00$ & $0.00 \pm 0.00$ & $0.00 \pm 0.00$ & $0.00 \pm 0.00$ & $25.00 \pm 0.00$ & $27.00 \pm 0.00$ & nd & nd \\
\hline S.pneumonia & $0.00 \pm 0.00$ & $23.00 \pm 0.00$ & $0.00 \pm 0.00$ & $0.00 \pm 0.00$ & $0.00 \pm 0.00$ & $6.00 \pm 0.00$ & $0.00 \pm 0.00$ & $37.00 \pm 0.00$ & nd & nd \\
\hline A.flavus & $12.00 \pm 0.00$ & $0.00 \pm 0.00$ & $10.00 \pm 0.00$ & $0.00 \pm 0.00$ & $0.00 \pm 0.00$ & nd & nd & nd & $28.00 \pm 0.00$ & $0.00 \pm 0.00$ \\
\hline C. albicans & $11.00 \pm 0.00$ & $12.00 \pm 0.00$ & $0.00 \pm 0.00$ & $0.00 \pm 0.00$ & $0.00 \pm 0.00$ & nd & nd & nd & $33.00 \pm 0.00$ & $0.00 \pm 0.00$ \\
\hline T.viridae & $0.00 \pm 0.00$ & $13.00 \pm 0.00$ & $10.00 \pm 0.00$ & $9.00 \pm 0.00$ & $10.00 \pm 0.00$ & nd & nd & nd & $39.00 \pm 0.00$ & $35.00 \pm 0.00$ \\
\hline
\end{tabular}

Each experiment was performed three times, and the data were averaged $(\mathrm{n}=3)$. Values are means of three replication \pm SD. Microorganisms: S. entomophilia $=$ Serratia entomophilia, E. caccae $=$ Entrecoccus caccae, P.sp $=$ Pseudomonas sp, E.coli $=$ Escherichia coli, L. acidophilus $=$ Lactobacillus acidophilus, $S$. pneumonia $=$ Streptococcus pnemonia, A. flavus $=$ Aspergillusflavus, C. albicans $=$ Candida albicans, . . viridae $=$ Trichophytonviridae . References $($ Positive control): Amp = Amplicillin, $\mathrm{Chl}=$ Chloramphenicol, Tet $=$ Tetracycline, Flu $=$ Fluconazole, Nys $=$ Nystatin. nd: not determine

The preliminary phytochemical screening revealed the presence of phenols, steroids, terpenoids, flavonoids, coumarins, alkaloids, saponins, tannins and the difference of secondary metabolites composition of fractions compared to crude extracts. This result can be explained by the fact that during fractionation, compounds are separated according to their affinity and solubility with extraction solvent (Cowan et al., 1999). However, the increasement of quantity of alkaloids and anthraquinones in methanol fraction compared to the loss of some classes of secondary metabolites in hexane and ethyl acetate fractions can be explain by the fact that fractionation could concentrated some classes of secondary metabolites and reduce others (Harbone, 1984; Bolou et al., 2011). The presence of all these classes of secondary metabolites in extract of root of L. mayombensis is an indication of its pharmacological importance. In fact, these classes of metabolites are reported to possess many importance biological activities including, antimicrobial, anticancer, antioxydant (Shariff, 2001; Ghoghari and Rajani, 2006; Hadacek, 2002; Panda and Kar, 2007). The fractionation of root extracts was lead to isolation and identification of 8 compounds and their antimicrobial activity was determined by disc diffusion method. The results showed that activity is closely related to structure of compounds tested. $\beta$-sitosterol and stigmasterol, respectively, two compounds of the same classes of steroids were found to target the same group of microorganisms. The antimicrobial activities of stigmasterol were found to be moderate as compared the reference drugs. This observation is in good agreement with previous reports that showed low antimicrobial (or antibacterial) activities of stigmasterol against Acetobacter sp., E. coli, S. aureus, and Streptococcus sp, P. aeruginosa (Ahamed et al., 2002; Tamokou et al., 2011). On the other hand, antibacterial activity of $\beta$ sitosterol was also found to be consistent with literature reports that discuss low/moderate antibacterial activity of $\beta$-sitosterol against several bacterial species that include $S$. aureus, E. coli, and $P$. aeruginosa (Beltrame et al., 2002). : (8S,9R,10R,11S,13R,17R)6,11-dihydroxy-14,15-epoxy-4,4,8,10,13-pentamethyl-17-(2-oxo2,5-dihydrofuran-3-yl-)-11,12,13,15,16,17-hexahydro -4Hcyclopent $[\alpha]$ phenanthrene-3,7(8H,9H,10H,14H)-dione (1) was the less active compounds studied. This can be explain the steric congestion around the hydroxyl group limiting his reactivity. The inhibitory activity of stigmasterol 3-O- $\beta$-D-glucopyranoside (8) was better than compounds $\mathbf{1 , 6}$ and $\mathbf{7}$. This activity was due to the substitution of the single and free hydroxyl on the carbon skeleton by the glucopyranoside group which enhance the activity of this stigmasterol. In fact, this glucopyranoside group is known as responsible for antimicrobial activity due to their ability to complex with bacteria cell wall, (Cowan, 1999) and therefore, inhibiting the microbial growth. The best and high spectrum antimicrobial activity was observed with 9,10-cyclopropyl$((3 S, 5 R, 8 R, 13 R, 14 S, 17 R) \quad)$-4,4,13,14,17-pentamethyl-17((2R,5S,6R)-5,6,7-trihydroxy-6-methylheptan-2-yl)-

hexadecahydro- $1 H$-cyclopentane $[\alpha]$ phenanthrèn-3-ol) (5) which is a cycloartane triterpenoïds. In fact, terpenoïds are involve in membrane disruption by the lipophilic compounds which lead to the death of microorganisms (Mendoza et al., 1997). The antimicrobial activities varied with the bacterial and fungal species. These variations may be due to genetic differences between the microorganisms. The findings of the present study showed that there were differences between the antimicrobial activities of isolated compounds. This suggests that the root extract of L. mayombensis contains several antifungal and antibacterial principles with different polarities as shown by the phytochemical study. Therefore, it is clear that limoinds and cycloartane are the active constituents of meliaceae family plant.

\section{ACKNOWLEDGEMENTS}

L.S.S. acknowledges the Centre for International Cooperation in Science (CICS) for a research grant INSA JRDTATA Fellowship (DO/CICS/4785/2014) at the Manonmaniam Sudaranar University.

\section{REFERENCES}

Ahamed MK, Krishna V, Gowdr HB, Rajanaika H, Kumaraswamy HM, et al. Isolation of bacterial constituents from the Stem Bark Extract of Grewia tiliaefoliaVahl. Res J Med Plant, 2007; 1: 72-82.

Amit R, Shailendra S. Limonoids: overview of significant bioactive triterpenes distributed in plants kingdom. Biol Pharm Bull, 2006; 29(2), 191-201. 
Bauer AW, Kirby WM, Sherris JC, Turck M. Antibiotic susceptibility testing by standardized single disk method. American Journal of Clinical Pathology, 1966; 45, 493-496.

Beltrame FL, Ferreira AG, Cortez DA. Coumarin glycoside from Cissus sicyoides. Nat Prod Lett, 2002; 16: 213-216.

Bolou GEK, Attioua B, N'guessan AC, Coulibaly A, N'guessan JD, Djaman AJ. Évaluation in vitro de l'activité antibactérienne des extraits de Terminalia glaucescens planch. sur Salmonella typhi et Salmonella typhimurium. Bulletin de la Société Royale des Sciences de Liège, 2011; 80: 772-790.

Cowan MM. Plant products as antimicrobial agents. Clin Microbiol Rev, 1999; 12: 564-582.

Hadacek F. Secondary metabolites as plant traits: current assessment and future perspectives. Crit Rev Plant Sci, 2002; 21, 273-322.

Harbone JB. 1973. Phytochemical Methods: a Guide to Modern Techniques of Plants Analysis. London: Chapman and Hall Ltd. pp. 50-116.

Harbone JB. 1984. Phytochemical Methods: A guide to modern techniques of plants analysis. $2^{\text {nd }}$ edition. Chapman and Hall, London. pp. $37-168$.

Harvey A. Strategies for discovering drugs from previously unexplored natural products. Drug Discov Today, 2000; 5(7):294-300.

Ghoghari AM, Rajani M. Densitometric determination of hecogenin Agave Americana leaf using HPTL. Chromatography, 2006; 64: 133-116.

Kai-Long J, Ping Z, Hua-Bin H, Shuai H, Shang-Gao L, YouKai X. Limonoids from the Leaves and Twigs of Walsura yunnanensis. J Nat Prod, 2014; 77, 1764-1769.

Khalijah A, Xe-Min L, Kok HL, Unang S, Marc L, Mat RM, Khalit M. Triterpenes and steroids from the leaves of Aglaia exima (Meliaceae). Fitoterapia, 2012; 83, 1391-1395.

Koumba Zaou P, Mapaga D, Nze Nguema S, Deleporte P. Croissance de 13 essences de bois d'oeuvre plantées en forêt Gabonaise. Bois et Forêts des Tropiques, 1998; 256(2): 21-32.

Li-Li W, Cheng-Shi J, Fei-Fei C, Hai-Yan Z, Yue-Wei G. Two New Limonoids from the Root Bark of Chinese Medicinal Plant Dictamnus dasycarpus. Helvetica Chimica Acta, 2014; 97, 1301-1306.

Mendoza L, Wilkens M, Urzua A. Antimicrobial study of the resinous exudates and of diterpenoids and flavonoids isolated from some Chilean Pseudogna phalium (Asteraceae). J Ethnopharmacol, 1997; 58:85-88.

Panda S, Kar A. Annonasqamosa seed extract in the regulation of hyperthyroidism and lipid-peroxidation in mice: Possible involvement of quercetin. Phytomedicine, 2007.14:799-805.
Rao MSA, Suresh G, Yadav PA, Prasad KR, Nayak VL, Ramakrishna S, Rao CV, Babu KS. Novel apo-tirucallane triterpenoids from Walsura trifoliata Tetrahedron Lett, 2012; 53, 6241-6244.

Nugroho AE, Okuda M, Yamamoto Y, Hirasawa Y, Wong CP, Kaneda T, Shirota O, Hadi, AHA, Morita H.. Walsogynes B-G, limonoids from Walsura chrysogyne. Tetrahedron, 2013; 69, 4139-4145.

Shariff ZU. 2001. Modern herbal therapy for common ailments. Nature Pharmacy Series (Volume1), Spectrum Books Limited, Ibadan, Nigeria in Association with Safari Books (Export) Limited, United Kingdom. pp. 9-84.

Tagatsing FM, Yankep E, Mbafor TJ, Atchade AT. Chemical constituents from stem barks of Millettia versicolor and Millettia zechiana. Rasayan J Chem, 2011; 4, 242-244.

Tan QG, Luo XD. Meliaceous Limonoids: Chemistry and Biological Activities Chem. Rev, 2011; 111, 7437-7522.

Tchouya, FGR, Bickii J, Barhé TA, Fekam BF, Djakou LB, Tchouankeu JC. Antiplasmodial activities of limonoids from Entandrophragma angolense (Meliaceae), and their semi synthetic derivatives: Study of the structure-activity relationship. Spatula DD, 2013; 3(2), 45-50.

Tamokou JD, Kuiate JR, Tene M, Julbelin T, Nwemeguela K, et al. The antimicrobial activities of extract and compounds isolated from Brillantaisia lamium. Iran J Med Sci, 2011; 36: 24-31.

Tsamo A, Langat MK, Nkounga P, Kamdem WAF, Nkengfack AE, Mulholland DA. Limonoids from the West African Trichilia welwitschii (Meliaceae). Biochemical Systematics and Ecology, 2013; 50, 368-370.

Verpoorte R. Exploration of nature's chemodiversity: the role of secondary metabolites as leads in drug development. Drug Discovery Today, 1998; 3, 232-238

Vieira IJC, Azevedo ODA, De Souza JJ, Braz-Filho R, Gonçalves MDS, De Araújo MF. Hirtinone, a Novel Cycloartane-Type Triterpene and Other Compounds from Trichilia hirta L. (Meliaceae). Molecules, 2013; 18, 2589-2597.

\section{How to cite this article:}

Sidjui LS, Tchangoue YAN, Radhakrishnan S, Karthiga P, Toghueo RMK, Famen LCN, Djomgoué P, Annadurai G, Folefoc GN. Preliminary in vitro antimicrobial screening of chemical constituents isolated from the root of Lepleae mayombensis (Meliaceae). J App Pharm Sci, 2015; 5 (12): 035-041. 